\title{
Tensor Network Methods for Invariant Theory
}

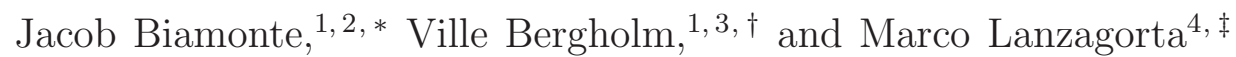 \\ ${ }^{1}$ Institute for Scientific Interchange, Via Alassio 11/c, 10126 Torino, Italy \\ ${ }^{2}$ Centre for Quantum Technologies, National University of \\ Singapore Block S15, 3 Science Drive 2, Singapore 117543 \\ ${ }^{3}$ Department of Chemistry, Technische Universität München, D-85747 Garching, Germany \\ ${ }^{4}$ US Naval Research Laboratory, 4555 Overlook Ave. SW, Washington DC 20375
}

Invariant theory is concerned with functions that do not change under the action of a given group. Here we communicate an approach based on tensor networks to represent polynomial local unitary invariants of quantum states. This graphical approach provides an alternative to the polynomial equations that describe invariants, which often contain a large number of terms with coefficients raised to high powers. This approach also enables one to use known methods from tensor network theory (such as the matrix product state factorization) when studying polynomial invariants. As our main example, we consider invariants of matrix product states. We generate a family of tensor contractions resulting in a complete set of local unitary invariants that can be used to express the Rényi entropies. We find that the graphical approach to representing invariants can provide structural insight into the invariants being contracted, as well as an alternative, and sometimes much simpler, means to study polynomial invariants of quantum states. In addition, many tensor network methods, such as matrix product states, contain excellent tools that can be applied in the study of invariants.

PACS numbers: 03.65.Fd, 03.65.Ud, 03.65.Aa, 03.67.-a

\footnotetext{
* jacob.biamonte@qubit.org

$\dagger$ ville.bergholm@iki.fi

$\ddagger$ marco.lanzagorta@nrl.navy.mil
} 
In quantum physics, polynomial invariants typically arise in the study of various entanglementrelated properties of quantum states [1 [6] and gates [7, 8]. In this paper we present a variant of the graphical tensor calculus of Penrose [9] for the purpose of representing and computing polynomial invariants of arbitrary quantum states.

The graphical approach of tensor network diagrams provides an alternative to the polynomial equations that describe invariants, which often contain a large number of terms with coefficients raised to high powers. It also enables one to use established methods from tensor network theory (such as matrix product state factorizations) to study polynomial invariants. We find in our examples that the underlying mathematical structure of the physics described by the invariants is reflected in the structure of the resulting tensor networks. By using specific graphical rewrite rules, our methods enable one to contract and simplify the tensor network representing any given polynomial invariant of a bipartite pure state to the point where the network is succinctly expressed in terms of Schmidt coefficients. This serves as a graphical proof of the invariance of the quantity represented by the network, as well as a conceptual aid geared towards understanding the meaning behind the invariants. The graphical method introduced to factor matrix product states is slightly different than known approaches in the literature - see for example, the matrix product states review [13] and [14, 15]. These differences are important for our purposes.

The area of tensor network states and tensor network algorithms is a rapidly growing area of physics which studies (in part) the most efficient way to represent quantum states and to discover key properties of quantum systems. One of the main methods inside this framework is the matrix product state (MPS) representation [13 17]. This method is not well known outside of physics. We find it to be well suited to study invariants and think that others working in the area of invariant theory (even outside of physics) will find the matrix product state factorization useful. We therefore hope that the present paper can help bridge this gap between these communities and foster further cross-pollination between invariant theory and tensor network states. For related work published after the preprint version of our study, see [18, 19].

At the heart of MPS is the tensor network description of repeated bipartitions of a quantum state. By capturing the singular value decomposition in a tensor network where all internal components have clearly defined algebraic properties, we present some small improvements in the graphical tensor calculus used to describe matrix product states, as well as invariants in general. In this regard, our results on matrix product states take an important first step in uniting invariant theory with tensor network states. The key example we consider here is showing the utility of tensor network methods for matrix product factorizations of quantum states into bipartitions.

Apart from the intuition found in representing states as MPSs, one might also employ tensor network algorithms [14, 15, 17, 20, 21] to design and contract invariants of interest to physics. Tensor network methods also offer a valuable conceptual aid to understanding how the numerical value of an invariant relates to properties of the state.

We begin by recalling the fundamental notions of the tensor calculus in Sections 1, 2] and 3 , This leads to the diagrammatic SVD, which is used in Section 4 to factor a given quantum state into a matrix product state. We then connect the tensor calculus to polynomial invariants in Section 5, Before concluding, we also consider the application of invariants to calculate entropies and entanglement measures.

\footnotetext{
${ }^{1}$ We assume readers are familiar with the basics of tensor networks, although we will review them in Section [1. Readers seeking the basics of tensor networks could consult 10 12] and those interested in tensor network algorithms and applications to physics could consult [13 15].
} 


\section{PENROSE GRAPHICAL NOTATION FOR TENSOR NETWORKS}

Penrose graphical notation [9] is a diagrammatic notation for tensor networks. This notation is becoming well known inside the tensor network algorithms community (see for example [22] for an early use of the graphical notation to describe matrix product states). It can make the manipulation of complicated tensor networks much easier and more intuitive. Contributions on the topic we found influential can be found in [23 27]. In our previous work, we have adapted the graphical notation and surrounding methods to describe generalized quantum circuits [11], tensor network states [10 12], open quantum systems [28, 29] as well as decidability in algorithms based on tensor contractions [30].

In the string diagram notation, a tensor is a graphical shape with a number of input legs (or "arms") pointing up, and output legs pointing down 2 Individual arms as well as individual legs each independently correspond to an index. For example,

(a)

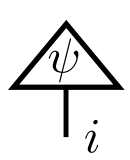

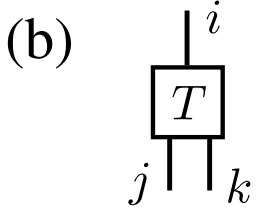

diagram (a) represents the tensor $\psi_{i}$ and (b) the tensor $T_{j k}^{i}$. A tensor with $n$ indices up and $m$ down is called a valence- $(n, m)$ tensor and sometimes a valence- $k$ tensor for $k=n+m$.

In quantum physics parlance one introduces a computational basis and expands the tensors in it; in which case $T_{j k}^{i}$ is understood not as abstract index notation but as the actual components of the tensor:

$$
T=\sum_{i j k} T_{j k}^{i}|j k\rangle\langle i| .
$$

In practice there is little room for confusion however.

There are three special "wire tensors" that play the role of the metric tensor 3 They are given diagrammatically as

(a)

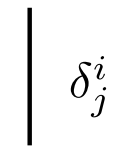

(b)

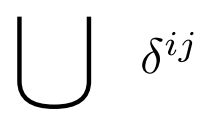

(c)

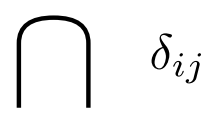

The identity tensor (a) is used for index contraction by connecting the corresponding legs, and the cup (b) and cap (c) are metric tensors used for raising and lowering indices. Expanding them in the computational basis we obtain

$$
\begin{aligned}
\mathbb{1} & =\sum_{i j} \delta^{i}{ }_{j}|j\rangle\left\langle i\left|=\sum_{k}\right| k\right\rangle\langle k|, \\
\langle\cup| & =\sum_{i j} \delta^{i j}\langle i j|=\sum_{k}\langle k k|, \quad \text { and } \\
|\cap\rangle & =\sum_{i j} \delta_{i j}|i j\rangle=\sum_{k}|k k\rangle .
\end{aligned}
$$

\footnotetext{
2 Often, to conserve space, the diagrams are rotated 90 degrees counterclockwise. In practice this should be obvious from the context.

${ }^{3}$ We will always work in a flat Euclidean space, which renders the metric tensors trivial.
} 
(a)

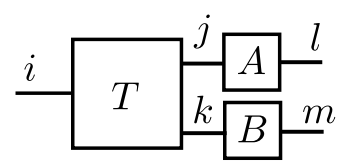

(b)

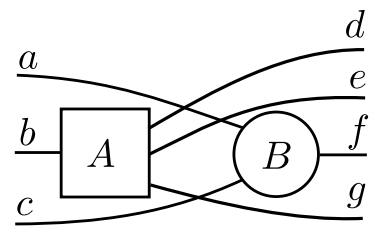

FIG. 1. Illustration of the graphical notation. (a) Contraction of tensor $T$ with tensors $A$ and $B$ amounts to joining indices: $T^{i}{ }_{j k} A^{j}{ }_{l} B^{k}{ }_{m}$. (b) Permutation of indices by crossing wires: $A^{b}{ }_{d e g} B^{a c}{ }_{f}$.

(i) One can raise and subsequently lower an index or vice versa, which amounts essentially to doing nothing at all. This scenario is captured diagrammatically by the so called snake or zig-zag equation

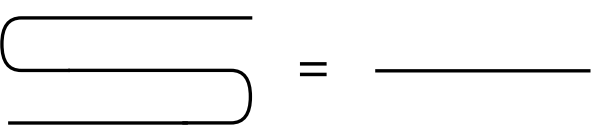

together with its mirror image [9]. In tensor index notation, it is expressed succinctly as $\delta^{i j} \delta_{j k}=\delta_{k}^{i}$.

(ii) Crossing two wires (as in diagram (a) below) is equivalent to swapping the relative order of the corresponding vector spaces.

(a)

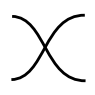

(b)

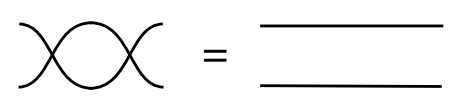

(b) illustrates that the swap operation is self inverse. It may be written as $\operatorname{SWAP}_{k l}^{i j}=$ $\delta^{i}{ }_{l} \delta^{j}{ }_{k}$.

(iii) The trace in the graphical calculus is given by appropriately joining wires to close loops.

Together the cups and caps give rise to a correspondence between different types of maps and states. We call the duality induced by bending and exchanging wires Penrose duality.

\section{PENROSE WIRE BENDING DUALITY}

Now we will consider the set of operations formed from bending tensor wires forwards or backwards using cups and caps, as well as exchanging wires using SWAP. We can conceptualize this set of transforms acting on a tensor as amounting essentially to matrix reshapes. From the snake equation, action with a cup or a cap is invertible and SWAP is self inverse. This implies that all possible configurations of a tensor's wires obtained using these operations can be considered equivalent. We will start with an example.

Example 1. Given a tensor $T^{i}{ }_{j}$ with fixed labels $i, j$ one uses cups and caps to rearrange index elevations, arriving at

$$
T^{i}{ }_{j}, T^{i j}, T_{i j}, T_{i}{ }^{j}
$$


Using the SWAP operation one reorders the horizontal position of $i$ and $j$. Then applying cups and caps yields

$$
T_{i}^{j}, T^{j i}, T_{j i}, T_{j}{ }^{i}
$$

for a total of eight possible reshapes.

For an $n$ index tensor, each index can be either up or down, yielding $2^{n}$ possibilities. The symmetry group formed by SWAP is of order $n$ ! and acts to arrange the horizontal position of the $n$ legs of a tensor, yielding (provided we distinguish forms of the type $T_{i}{ }^{j}$ and $T_{i}^{j}$ in Example 1) $n ! \cdot 2^{n}$ different ways to reorder the $n$ indices of a tensor.

Remark 2 (Ordering operators by numbers of inputs and outputs). In the previous example, we considered $T_{i}^{j}$ (b) and $T_{i}^{j}$ (a) as distinct. This is illustrated in (a) and (b) as follows.

(a)

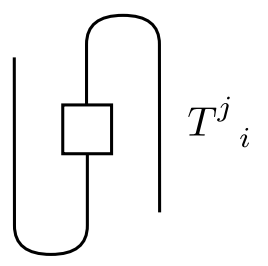

(b)

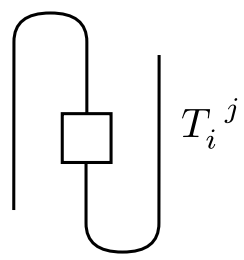

This provides an example of an awkward property of the inherently one-dimensional Dirac notation. Both (a) and (b) represent the same map, but when we write them in a basis, consistency dictates that one will expand in the basis $\langle i|\otimes| j\rangle$.

With the equivalence explained in Remark 2 in mind, we note that the tensor $T^{i}{ }_{j}$ from Example 1 actually has six unique reshapes, as two pairs of reshapes are diagrammatically equivalent. In other words, in (b) below we have that $T_{j}^{i}=T^{i}{ }_{j}=T_{j}{ }^{i}$ and for (e) we have the equality $T_{i}^{j}=T_{i}^{j}=T_{i}{ }^{j}$.

(a)

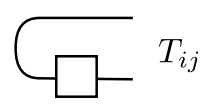

(d)

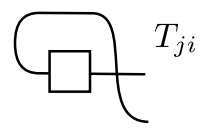

(b)

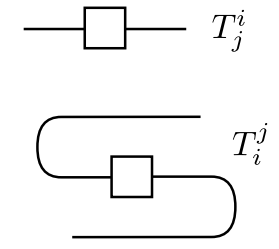

(c)

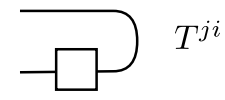

(f)

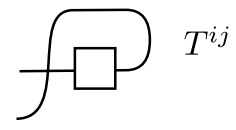

Together, we call these reshapes the natural tensor symmetry class. More generally one finds the number of diagrammatically unique reshapes of a tensor by (i) counting the number of possible ways it can have its wires bent, either forward or backwards using the cups and caps, and (ii) the number of ways a tensor can have its arms and/or legs reordered. We arrive at the following result:

Theorem 3 (Natural tensor symmetry class). The arms and legs of a tensor $\Gamma_{q r \cdots s}^{i j \cdots k}$ with $n$ input and output legs in total can be rearranged in $(n+1)$ ! different ways.

\section{DIAGRAMMATIC SVD}

In this section, we introduce the diagrammatic representation of the singular value decomposition (SVD). Later it will be used to simplify invariants obtained through network contraction, and iterated to obtain a matrix product state (MPS) description for a pure state. 
The SVD factors tensors into well defined building blocks with simplistic interaction properties: (i) a valence-one tensor storing singular values, (ii) a valence-three-COPY tensor used to create a diagonal map, and (iii) a pair of valence-two unitary gates. COPY-tensors have been studied in the setting of the Penrose tensor calculus, in work dating back at least to Lafont [23, 24] — see also [10, 11, 25].

Definition 4 (COPY tensor). The $m$-to- $n$ COPY tensor is defined in the computational basis as

$$
\operatorname{COPY}_{m \rightarrow n}:=\sum_{k=0}^{d-1}|\underbrace{k \cdots k}_{n}\rangle\langle\underbrace{k \cdots k}_{m}| .
$$

It is named accordingly because connecting a basis state $|k\rangle$ to any of its input or output wires collapses the sum and breaks the tensor up into unconnected copies of $|k\rangle$ and $\langle k|$. As with classical circuits, in the diagrammatic tensor notation COPY $m \rightarrow n$ is represented by a simple black dot $\bullet$ with $m$ input and $n$ output legs. Since all the legs of a COPY tensor are identical, and the inputs can be converted to outputs and vice versa simply by using cups and caps, keeping track of the direction of the legs is not important as long as they are connected to other tensors. This is reflected in the notation. For a brief enumeration of the algebraic properties of the COPY tensor, see [11].

Theorem 5 (Diagrammatic SVD). Any valence-two tensor $f: A \rightarrow B$ can be factored into a non-negative, unique valence-one tensor $\Sigma$, a valence-three COPY tensor, unitary valence-two tensors $U$ and $V$, and a diagonal valence-two dimension changer tensor $Q$ when necessary:

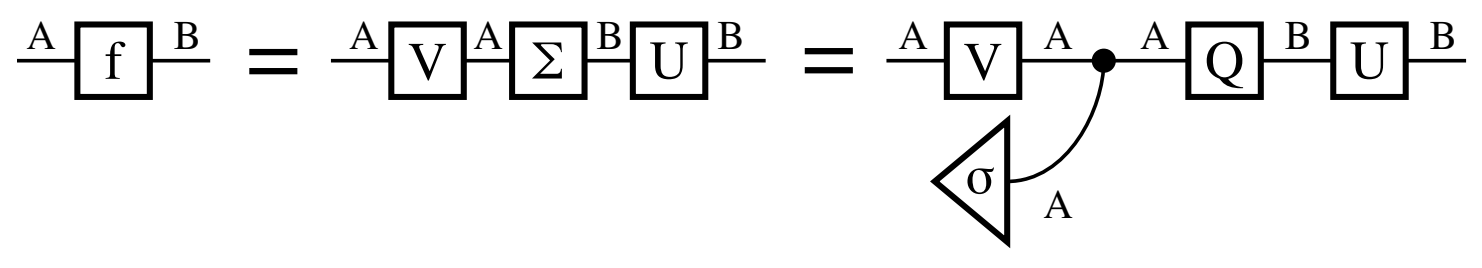

The dimension changing tensor $Q: A \rightarrow B$ has 1's on the diagonal and zero entries otherwise.

Proof. The SVD of $f$ is

$$
f=U \Sigma V,
$$

where $U: B \rightarrow B$ and $V: A \rightarrow A$ are unitary operators and $\Sigma: A \rightarrow B$ is diagonal in the computational basis, with the (necessarily non-negative) singular values $\sigma_{i}$ of $f$ along the diagonal. $\Sigma$ can be written as

$$
\Sigma=\sum_{j=0}^{d-1} \sigma_{j}|j\rangle_{B}\langle\left. j\right|_{A}=\underbrace{\sum_{i=0}^{d-1}|i\rangle_{B}\left\langle\left. i\right|_{A}\right.}_{Q_{A B}} \underbrace{\sum_{j}|j\rangle_{A}\left\langle\left. j j\right|_{A}\right.}_{\mathrm{COPY}_{2 \rightarrow 1}} \underbrace{\sum_{k} \sigma_{k}|k\rangle_{A}}_{\sigma} \quad\left(\sigma_{k} \geq 0\right),
$$

where $d=\min (\operatorname{dim} A, \operatorname{dim} B)$. We have expressed $\Sigma$ as a contraction of an valence-one tensor $\sigma$ with a COPY tensor. The non-square tensor $Q_{A B}$ is only necessary if $A$ and $B$ have different dimensions. 
Corollary 6 (Diagrammatic Schmidt decomposition). Given a bipartite state $|\psi\rangle \in A \otimes B$, we use the snake equation to convert it into a linear map $f: A \rightarrow B$ (inside the dashed region below) 4

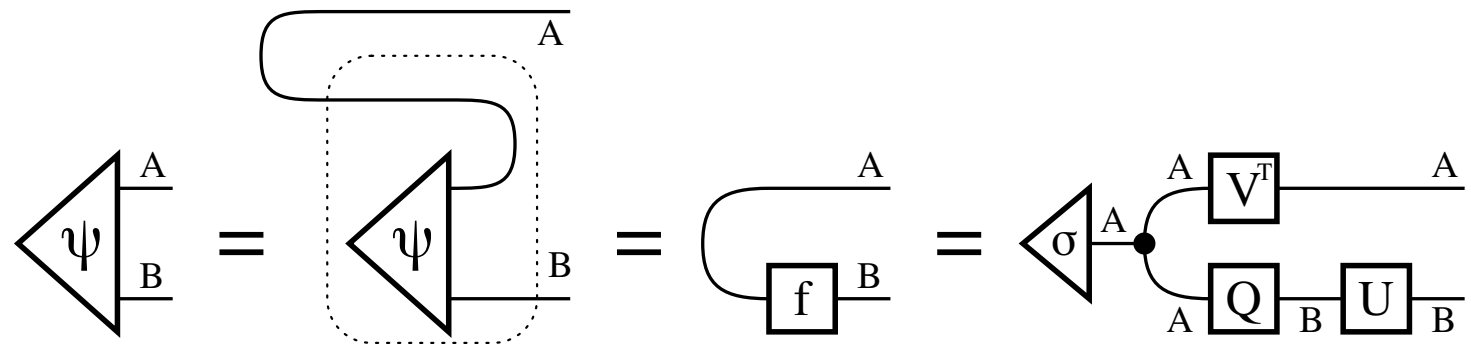

Now we apply the SVD as in Theorem 5. Diagram reorganization leads to the diagrammatic Schmidt decomposition of $|\psi\rangle$. The singular values in $\sigma$ now correspond to the Schmidt coefficients.

The network topology of the diagrammatic Schmidt decomposition can be used to study the entanglement properties of the bipartite state $|\psi\rangle$.

Example 7 (Entanglement topology). The topology of a bipartite state $|\psi\rangle=\sum_{i} \sigma_{i}\left|\varphi_{i}\right\rangle\left|\phi_{i}\right\rangle$ depends on the singular values in the triangular tensor $|\sigma\rangle=\sigma_{0}|0\rangle+\sigma_{1}|1\rangle+\ldots+\sigma_{d-1}|d-1\rangle$.

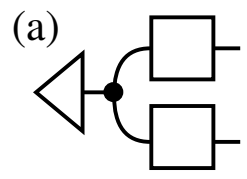

(b)

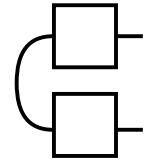

(c)

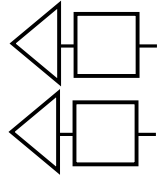

The general diagram state (a) takes the form (b) iff the Schmidt coefficients all have the same value. Now $|\sigma\rangle$ is proportional to a unit for the COPY-tensor, and the tensor structure is converted to a smooth wire, yielding the maximally entangled case. The most significant topology change (c) occurs when the input state to the COPY tensor is a single basis state $|\sigma\rangle=|0\rangle$. As this is a copy-point for the COPY-tensor, it breaks into two copies of $|\sigma\rangle$ and separates the diagram into two halves, illustrating the fact that the state is factorizable.

\section{DIAGRAMMATIC MATRIX PRODUCT STATES}

We will now consider matrix product states (MPS), an iterative method to factor quantum states into a linear chain of tensors (see [14, 15, 31] for a recent review and [11] for work considering the category theory behind MPS). The reason this factorization is called a 1D method is because it is known to describe a class of 1D systems efficiently, and because the factorization results in a 1D chain (for a discussion of other factorizations and the connection to geometry see for instance [31]). Without loss of generality, we will apply the MPS method to a four-party state, and explain the procedure in terms of three distinct steps.

Remark 8 (Method summary). MPS correspond to an iterative factorization method for quantum states. The key idea is a recursive application of the singular value decomposition

4 This has also been understood as a diagrammatic form of map-state duality underlying bipartite entanglement evolution [29]. 
(SVD). It begins by first selecting a bipartition and then applying the SVD. If either of the initial bipartitions can themselves be bipartitioned, the SVD is applied again. This results in a 1D tensor network representation of the state, as described in further detail below.

Consider a quantum state, expressed as a triangle in the Penrose graphical notation with a label 1 inside and open legs labeled $i, j, k, m$.

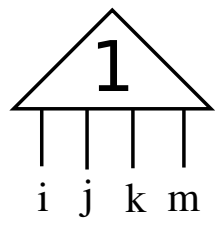

(Step I). We will create a bipartition comprising a first collection, containing only leg $i$ and a second, containing legs $j, k, m$. We will then apply the diagrammatic SVD across this partition. The partition is illustrated with the dashed cut below in (a). Diagram (b) results from applying the diagrammatic SVD across this partition, factoring the original state labeled $\mathbf{1}$ into a valence-two unitary box labeled $\mathbf{2}$, a valence-one triangle containing the singular values labeled $\mathbf{3}$, and a valence-four triangle labeled $\mathbf{4}$, all contracted with a COPY-tensor, as illustrated. A new internal label (d) for the wire connecting the COPYtensor to the valence-four triangle (4) was introduced for clarity. See also Fig. 2(a,b).

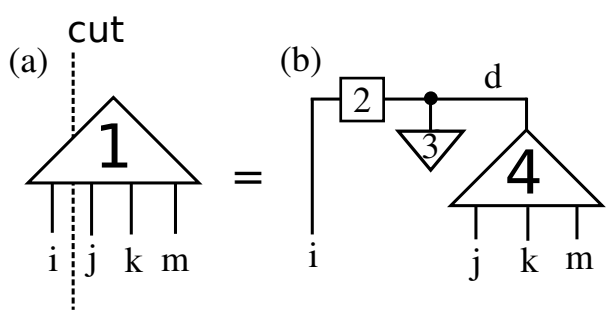

Remark 9 (Isometric internal tensors). The valence-four triangle tensor labeled 4 above arises from contracting a unitary map with a dimension changing tensor $Q$ (see (a) below). The input leg shown is labeled d. The other legs are contracted with a fixed basis state $|0\rangle$, from the SVD in (a) above. From the unitarity property, the isometry property follows, as illustrated graphically in (c) and (d) below.

(a)

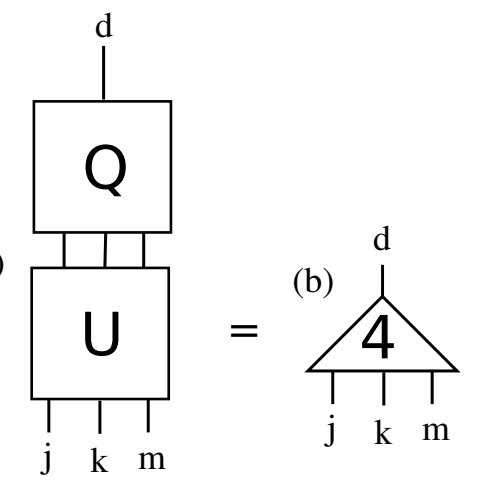

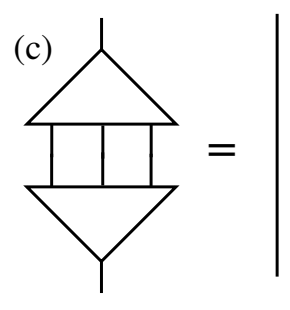

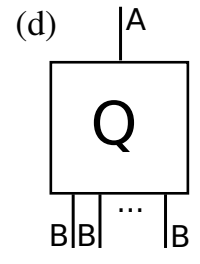

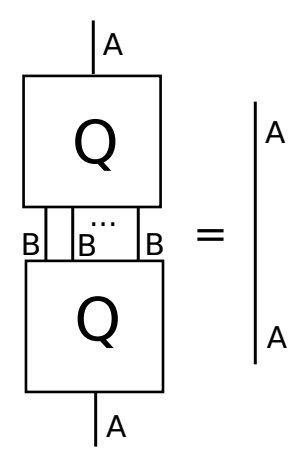

(Step II). To illustrate the next step in the factorization, we will remove the tensor labeled 4 by breaking the wire connecting it to the COPY-tensor (a). We will then partition this separate tensor into two halves, one containing wires $d, j$ the other half wires $k, m$. This 


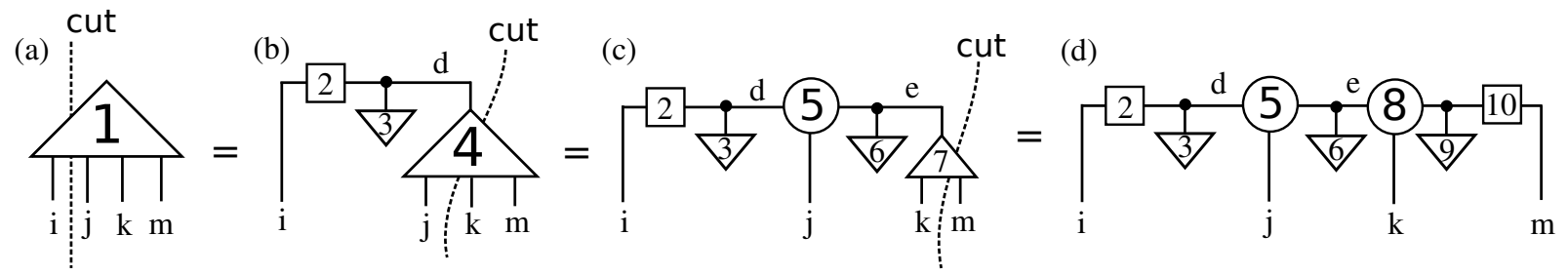

FIG. 2. MPS factorization steps (diagrammatic description of steps I, II and III). The quantum state (a) is iteratively factored into the $1 \mathrm{D}$ matrix product state $(\mathrm{d})$. This procedure readily extends to $n$-body states.

partition is illustrated by placing a dashed line (labeled cut) in (a). We arrive at the structure in (b), which we have explained in the first step. See also Fig. 2(b,c).

(a)

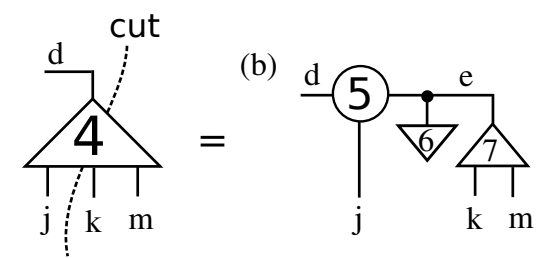

Remark 10 (An elementary property of tensor network manipulation). It is a fundamental property of tensor network theory that one can remove a portion of a network, alter this removed portion of the network without changing its function, and replace it back into the original network, leaving the function of the original network intact.

(Step III). In the third and final step of the MPS factorization applied to this four-party example, following remark 10 we first place the tensor we have factored in the second step, back into the original network from the first step, see (a) below. We then repeat the second step, applied to the triangular isometry tensor, labeled internally with a 7 . This results in the factorization appearing in (b). See also Fig. 2 (c,d).

(a)

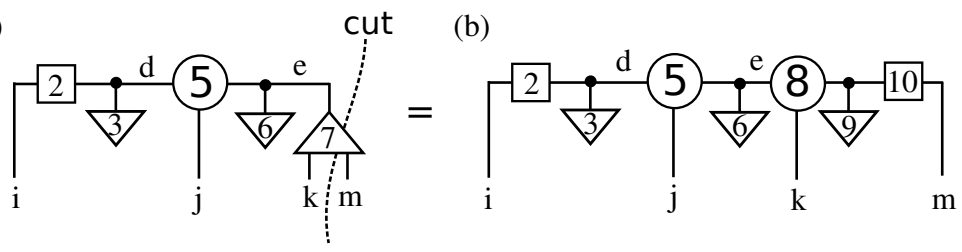

The iterative method continues in the same fashion as the first three steps, resulting in a factorization of an $n$-party state. A summary of the MPS factorization applied to a fourparty state is shown in Figure 2.

(Summary). We will now consider Figure 3, which summarizes the factorization scheme. In the steps we have outlined, we have factored Figure 3(a) into the MPS in Figure 3(d), in terms of the components listed below.

(i) States (labeled 3, 6 and 9; denoted $\phi_{3}, \phi_{6}$ and $\phi_{9}$, respectively): $\phi_{3}=\left(\lambda_{0}, \lambda_{1}\right), \phi_{6}=$ $\left(\lambda_{2}, \lambda_{3}, \lambda_{4}, \lambda_{5}\right)$ and $\phi_{9}=\left(\lambda_{6}, \lambda_{7}\right)$. The $\lambda_{i}$ 's are the singular values across each partition. The number of non-zero singular values $(\chi)$ is given by the minimum dimension of the two halves from the cut. For the case of qubits, the first outside partition has at most two non-zero entries, and the next inside partition has at most four. One might also 
(a)

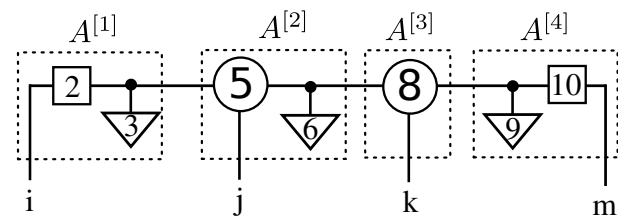

(b)

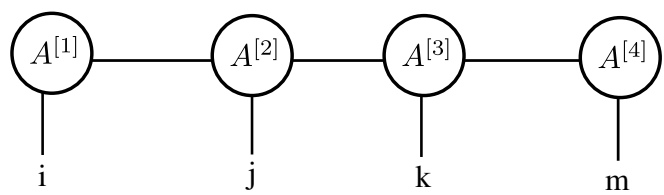

FIG. 3. Conversion from our notation (a), to conventional MPS notation (b). The factorization methods we have presented here and elsewhere [10, 11] allow one to "zoom in" and expose internal degree of freedom (a) or "zoom out" and expose high-level structure (b). The equational representation of the MPS in (b) is given in (24).

consider the singular values as the square roots of the eigenvalues of either member of the pair of reduced density matrices found from tracing out either half of a partition.

(ii) Unitary gates (labeled 2 and 10; denoted $U_{2}$ and $U_{10}$, respectively).

(iii) Isometries (labeled 5 and 8 ; denoted $I_{5}$ and $I_{8}$, respectively). The isometry condition describes the tensor relation $I_{j q}^{d} \bar{I}_{r}^{j q}=\delta^{d}{ }_{r}$. It is a consequence of the fact that tensors $I_{5}$ and $I_{8}$ arise from unitary gates, as explained in Remark 9, The isometry condition plays a more relevant role in structures other than 1D tensor chains.

We note that by appropriately combining neighboring tensors as in Figure 3(a), one recovers the familiar matrix product representation of quantum states 3(b). Matrix product states are written in equational form as

$$
|\psi\rangle=\sum_{i j k m} A_{i}^{[1]} A_{j}^{[2]} A_{k}^{[3]} A_{m}^{[4]}|i j k m\rangle .
$$

Here $A^{[1]}$ becomes a new tensor formed from the contraction of tensors labeled $\mathbf{2}, \mathbf{3}$, and $A^{[2]}$ is a contraction of tensors labeled $\mathbf{5}$ and $\mathbf{6}$, etc.

Remark 11 (Freedom in the representation). Readers would have noticed that we made a choice to perform the factorization starting from the left of the tensor and applying the SVD successively on tensors as we moved to the right. This apparent ambiguity has been characterized in detail [17]. It corresponds to a gauge freedom (on internal wires) given by action of the special linear group where the dimension of the representation is given by the dimension of the wires (e.g. the internal bond dimension). For open boundary conditions as has been considered here, there is a "canonical gauge" given first by Vidal. It is unique up to degeneracies in the spectrum of local reduced density operators [17].

A utility of our approach summarized in Figure 3(a) is that the COPY-tensor is well defined in terms of purely graphical rewrite identities (as seen in Definition 4). These graphical relations allow one to gain insights (into e.g. polynomial invariants as will be seen). The factorization we present however, allows one to preform many diagrammatic manipulations with ease, and exposes more structure inherent in a MPS.

Remark 12 (Data compression). The compact representation of a MPS is recovered by picking a cutoff value for the singular values across each partition, or a maximum number of allowed singular values. This allows one to compress data by truncating the Hilbert space and is at the heart of MPS computer algorithms in current use.

The singular values found from the MPS factorization can be used to form a complete polynomial basis to express invariant quantities related to an MPS. 


\section{PENROSE NOTATION MEETS ENTANGLEMENT INVARIANTS}

Here we will consider the variant of the graphical tensor calculus of Penrose [9] we have tailored to represent and contract polynomial invariants. We must first recall the notions surrounding polynomial invariants.

\section{Polynomial invariants}

Assume we are given a group $G$, a vector space $V$, and a group representation $D: G \rightarrow$ Aut $(V) 5$ Given a set $Q$, a function $f: V \rightarrow Q$ is an invariant function or simply an invariant under $D$ iff it is constant on the orbits of $D 6$

In the context of quantum mechanics, the vector space $V$ is typically either the state space $\mathcal{H}$, or $\operatorname{End}(\mathcal{H})$, the space of linear operators $\mathcal{H} \rightarrow \mathcal{H}$. Any representation $D: G \rightarrow \operatorname{Aut}(\mathcal{H})$ on $\mathcal{H}$ induces a representation $R: G \rightarrow \operatorname{Aut}(\operatorname{End}(\mathcal{H}))$ on $\operatorname{End}(\mathcal{H})$ :

$$
R(g)(\rho)=D(g) \rho D^{-1}(g) .
$$

An important class of invariants are the polynomial invariants $f: V \rightarrow \mathbb{C}$, which are polynomial functions of the coefficients of $\rho$ or $|\psi\rangle$ in the standard basis. The study of such polynomials is known as invariant theory [32]. David Hilbert made notable progress on this topic, which he pursued throughout his life. There has been past work considering these invariants in the context of quantum information science. Some that was influential to us includes [1 [5]. See also the complementary recent study [18].

Remark 13 (Basis independence). To form a polynomial out of the coefficients of a state, one first chooses a basis to express the state in. The value of the polynomial generally depends on the basis chosen. However, a polynomial that is invariant under any group that contains the local unitary group as a subgroup is inherently basis independent as long as our basis is a tensor product of orthonormal local bases.

\section{Invariance under the local unitary group}

Definition 14 (Local unitary (LU) equivalence of states). Two quantum states (pure or mixed) in the Hilbert space $\mathcal{H}=\mathcal{H}_{1} \otimes \mathcal{H}_{2} \otimes \ldots \otimes \mathcal{H}_{n}$ are LU equivalent iff they are related by a local unitary transformation, that is, a member of the natural representation of the group

$$
G_{\mathrm{LU}}:=U(1) \times S U\left(d_{1}\right) \times S U\left(d_{2}\right) \times \ldots \times S U\left(d_{n}\right),
$$

where $d_{i}=\operatorname{dim} \mathcal{H}_{i}$ is the dimension of the $i$ th subsystem. LU equivalence yields a partitioning of the state space into LU orbits. Entanglement measures are by definition LU invariants, i.e., constant on the aforementioned equivalence classes.

We now present a diagrammatic method for systematically generating polynomial LU invariants for state vectors and operators by casting the method of Grassl et al. [1, 33] into a form based on the Penrose tensor calculus. The method generates homogeneous polynomials

$5 \operatorname{Aut}(V)$ denotes the group of automorphisms of $V$, i.e. the invertible linear maps from $V$ to itself.

6 Or equivalently iff $f$ itself is a fixed point under the induced representation $D^{\prime}: G \rightarrow \operatorname{Aut}(F(V, Q))$, $D^{\prime}(g)(f)=f \circ D\left(g^{-1}\right)$. 
in the state coefficients that are necessarily invariants of the local unitary group 7 A utility of generating this set stems from the fact that the tensor networks considered can be used to calculate quantities that are invariant under the action of the local unitary group.

Given a density operator $\rho: \mathcal{H} \rightarrow \mathcal{H}$, consider the network

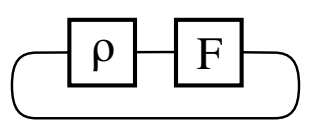

equivalent to the expression $\operatorname{Tr}(F \rho)=F^{i}{ }_{j} \rho_{i}{ }_{i}$. By choosing a suitable $F$, we can represent any first-degree homogeneous polynomial in the coefficients of $\rho$ in this way. Likewise, the tensor network

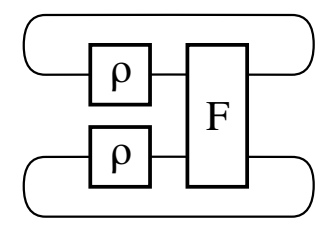

translates to $\operatorname{Tr}\left(F \rho^{\otimes 2}\right)=F^{i l}{ }_{j k} \rho_{i}^{j} \rho_{l}^{k}$, giving all the second-degree homogeneous polynomials. The procedure carries on in this fashion. A diagram with $k$ copies of $\rho$ gives us all the homogeneous polynomials of degree $k$ :

$$
\operatorname{Tr}\left(F \rho^{\otimes k}\right)=F_{p q \cdots t}^{i j \cdots m} \rho_{i}^{p} \rho_{j}^{q} \cdots \rho_{m}^{t} .
$$

Having thus generated a complete basis for the polynomials in the coefficients of $\rho$, we next wish to find out which of these homogeneous polynomials are invariant under the natural representation of $G_{\mathrm{LU}}$ :

$$
\operatorname{Tr}\left(F\left(U \rho U^{-1}\right)^{\otimes k}\right)=\operatorname{Tr}\left(\left(U^{-1}\right)^{\otimes k} F U^{\otimes k} \rho^{\otimes k}\right)=\operatorname{Tr}\left(F \rho^{\otimes k}\right) \quad \forall U \in G_{\mathrm{LU}}, \quad \forall \rho .
$$

This is fulfilled iff

$$
\left[F, U^{\otimes k}\right]=0 \quad \forall U \in G_{\mathrm{LU}} .
$$

We are then faced with finding matrices $F$ that commute with $U^{\otimes k}$ for each $U \in G_{\mathrm{LU}}$. The solution is roughly stated in the following theorem.

Theorem 15 (Brauer [34], Procesi [33]). The algebra of matrices that commute with every $U^{\otimes k}$ for $U \in G_{L U}$ is generated by the unitary representation

$$
T: \underbrace{S_{k} \times \ldots \times S_{k}}_{n \text { copies }} \rightarrow \text { Aut } \mathcal{H}^{\otimes k}
$$

of the $n$-fold direct product of the permutation group $S_{k}$ which, independently for each of the $n$ subsystems, permutes the relative ordering of the $k$ copies of that subsystem's state space within the total space $\mathcal{H}^{\otimes k}$.

\footnotetext{
${ }^{7}$ Although we can generate a complete set of invariants in this fashion, except in rare cases, finding a minimal complete set of polynomial invariants is computationally difficult. This alternative line of research has been a key focus in the connection of invariant theory with quantum entanglement.
} 
Hence, it is enough to consider matrices $F$ which correspond to these permutation maps. The permutation group has a well known and evident diagrammatic form. Below, we show the elements of the permutation group (a) $S_{1}$, (b) $S_{2}$, and (c) $S_{3}$.

(a)

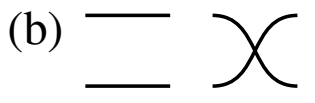

(c)

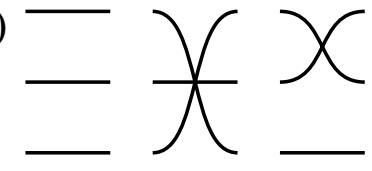

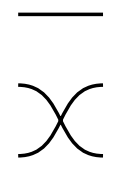
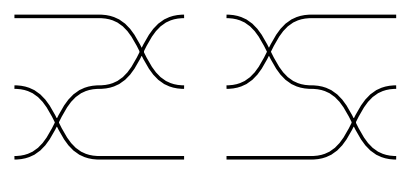

We then carry on to evaluate all the expressions of the form

$$
I_{k ; \sigma_{1}, \sigma_{2}, \ldots, \sigma_{n}}(\rho):=\operatorname{Tr}\left(T\left(\sigma_{1}, \sigma_{2}, \ldots, \sigma_{n}\right) \rho^{\otimes k}\right), \quad \text { where } \sigma_{i} \in S_{k}
$$

to generate the homogeneous invariant polynomials of degree $k$ 专 It is easy to see that the diagrams we obtain are indeed invariant under $G_{\mathrm{LU}}$, as shown in Figure 4 .

(a)

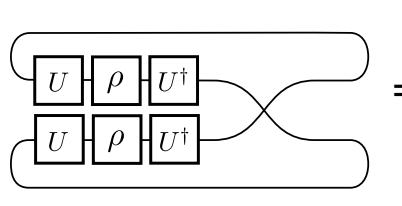

(b)

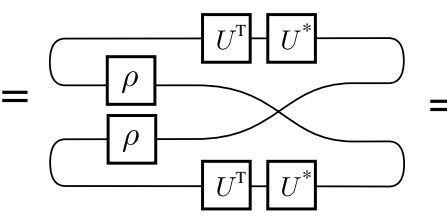

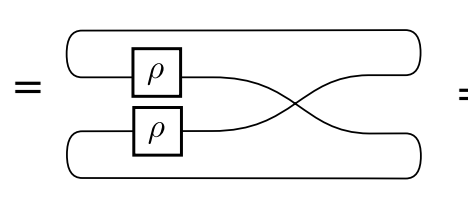

(c)

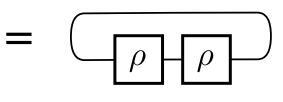

FIG. 4. Proof of the invariance of $I_{2 ;(12)}$. Having acted on $\rho$ with some unitary operation $U$, we slide $U$ and $U^{\dagger}$ around the bends, taking the transpose and resulting in (b). The unitaries cancel and the diagram reduces to $(\mathrm{c})$, showing that it indeed describes an invariant. A little bit of further manipulation shows that $I_{2 ;(12)}$ evaluates to $\operatorname{Tr}\left(\rho^{2}\right)$.

Not all of these invariants are independent, or even distinct. We can eliminate some of the redundancy using the following theorem, proven in [1]:

Theorem 16 (Invariant distinctness [1]). Since all the copies of $\rho$ in (34) are identical, we may permute their relative order without changing the invariant. This is equivalent to conjugating each subsystem permutation $\sigma_{i}$ with the same element $\tau \in S_{k}$ :

$$
I_{k ; \sigma_{1}, \ldots, \sigma_{n}}=I_{k ; \tau \sigma_{1} \tau^{-1}, \ldots, \tau \sigma_{n} \tau^{-1}} \quad \forall \sigma_{i}, \tau \in S_{k} .
$$

This theorem enables us to arrange each invariant diagram to the following canonical form which makes it easy to tell if two diagrams are topologically distinct.

(i) The $k$ copies of the system are arranged such that the permutation on the first subsystem is grouped by cycles, ordered by non-increasing cycle length.

(ii) The process is repeated on the second, then third etc. subsystem within the remaining permutational freedom, i.e. cyclic permutation within the cycles and permuting cycles of identical length.

If a particular diagram is not connected, the corresponding invariant is the product of the invariants corresponding to the disjoint sub-diagrams.

8 We use the cycle notation to denote specific elements $\sigma$ of the permutation groups. 
Remark 17. Note that using the procedure here, two algebraically independent invariants necessarily have topologically distinct diagrams but the converse does not necessarily hold.

Theorem 18 (Real-valuedness of the invariants). If all the permutations $\sigma_{i}$ are self-inverse, or can all be inverted by conjugating them with the same element $\tau$ as shown in Theorem 16, the invariant $I_{k ; \sigma_{1}, \ldots, \sigma_{n}}$ is necessarily real for all states.

Proof.

$$
\begin{aligned}
I_{k ; \sigma_{1}, \ldots, \sigma_{n}}^{*}(\rho) & =\operatorname{Tr}\left(\left(\rho^{\otimes k}\right)^{\dagger} T^{\dagger}\left(\sigma_{1}, \ldots, \sigma_{n}\right)\right)=\operatorname{Tr}\left(T^{\dagger}\left(\sigma_{1}, \ldots, \sigma_{n}\right) \rho^{\otimes k}\right) \\
& =\operatorname{Tr}\left(T\left(\sigma_{1}^{-1}, \ldots, \sigma_{n}^{-1}\right) \rho^{\otimes k}\right)=I_{k ; \sigma_{1}^{-1}, \ldots, \sigma_{n}^{-1}}(\rho) .
\end{aligned}
$$

Theorem 19 (States with a single subsystem). All the independent invariants of a $d$ dimensional state $\rho$ with a single subsystem are given by

$$
I_{k}:=\operatorname{Tr}\left(\rho^{k}\right), \quad \text { where } k \in\{1,2, \ldots, d\} .
$$

Proof. The only degree one invariant, $I_{1}:=\operatorname{Tr}(\rho)$, is presented in Figure 5. The two possible degree two invariants are shown in Figure 5. b. The first one is simply $I_{1}^{2}$. The second one, $I_{2}:=\operatorname{Tr}\left(\rho^{2}\right)$, however is independent. Likewise, the only independent invariant of degree three, $I_{3}:=\operatorname{Tr}\left(\rho^{3}\right)$, is given in Figure 5.c. In general, at each degree $k$ we obtain a single new independent invariant $I_{k}:=\operatorname{Tr}\left(\rho^{k}\right)$, by using a complete permutation connecting all the $k$ copies of the state.

(a)

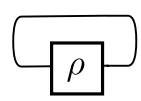

(b)

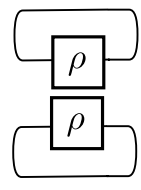

(c)

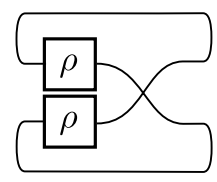

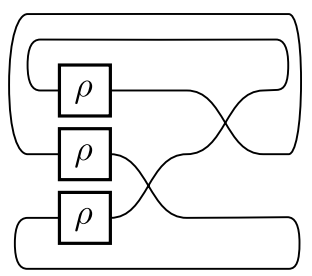

FIG. 5. LU invariants for a single subsystem. (a) $I_{1}:=I_{1 ; e}=\operatorname{Tr}(\rho)$. (b) $I_{2 ; e}=I_{1}^{2} \cdot I_{2}:=I_{2 ;(12)}=$ $\operatorname{Tr}\left(\rho^{2}\right)$. (c) $I_{3}:=I_{3 ;(123)}=\operatorname{Tr}\left(\rho^{3}\right)$.

The Cayley-Hamilton theorem now tells us that the basis is finitely generated, as every $\rho$ satisfies its own characteristic polynomial, giving an $d$ th degree polynomial equation in $\rho$ which enables us to express any $I_{m}$ with $m>d$ in terms of the lower-degree invariants [33].

Example 20 (Invariants for a single qubit). The only independent (fundamental) invariants of a single qubit state are $I_{1}$ and $I_{2}$, defined in the previous theorem. $I_{1}=\operatorname{Tr}(\rho)$ is the norm of the state. $I_{2}=\operatorname{Tr}\left(\rho^{2}\right)$ turns out to be precisely the purity of the state. In terms of the eigenvalues $\left(\lambda_{0}, \lambda_{1}\right)$ of $\rho$ we have $I_{1}=\lambda_{0}+\lambda_{1}=1$ (for normalized states) and $I_{2}=\lambda_{0}^{2}+\lambda_{1}^{2}$. From the Cayley-Hamilton theorem we have that there is a second degree monic polynomial in $\rho$ that vanishes identically. In other words, constants $a, b$ exist such that

$$
\rho^{2}+a \rho+b \mathbb{1}=0 .
$$


Multiplying both sides by $\rho^{m}$ and taking the trace, we obtain the recurrence relation

$$
I_{m+2}+a I_{m+1}+b I_{m}=0
$$

and thus find that the traces of higher powers of $\rho$ can be expressed in terms of $I_{1}$ and $I_{2}$. These invariants are indeed algebraically independent and complete, meaning any other polynomial invariant can be expressed in $\left\{\mathbb{R},+, \cdot, I_{1}, I_{2}\right\}$. For instance,

$$
\operatorname{det}(\rho)=\frac{1}{2}\left(\operatorname{Tr}(\rho)^{2}-\operatorname{Tr}\left(\rho^{2}\right)\right)=\frac{1}{2}\left(I_{1}^{2}-I_{2}\right)=\lambda_{0} \lambda_{1}
$$

Likewise, $I_{3}=\lambda_{0}^{3}+\lambda_{1}^{3}$ can be written as

$$
I_{3}=I_{1}\left(I_{2}-\operatorname{det}(\rho)\right)
$$

Remark 21 (Bipartite states). For bipartite states we obtain a much more complicated set of invariants. Figure 6 presents all the topologically distinct invariants up to $k=3$.

(a)

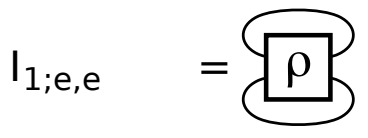

(b)

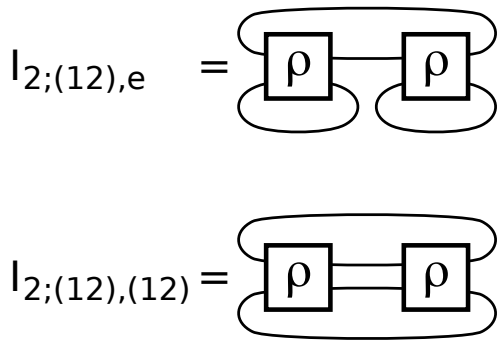

(c)

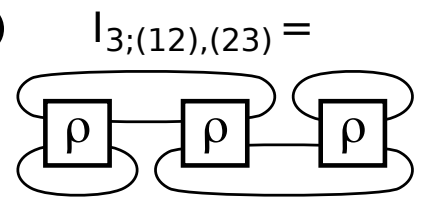

$\mathrm{I}_{3 ;(123), \mathrm{e}}=$

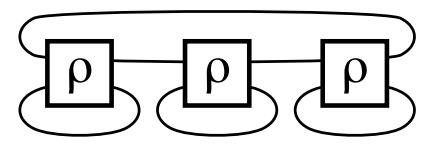

$\mathrm{I}_{3 ;(123),(12)}=$

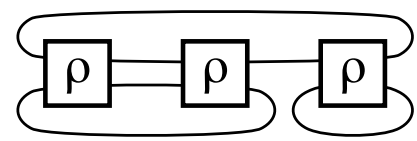

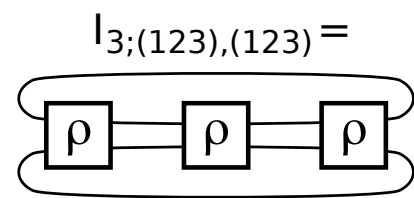

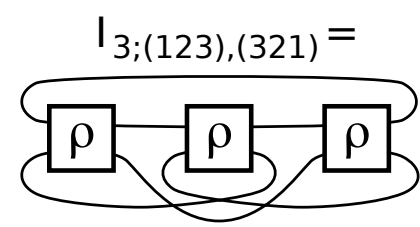

FIG. 6. LU invariants of a bipartite system up to $k=3$. To avoid listing essentially similar diagrams we only show here the distinct invariants modulo swapping the order of the two subsystems. (a) The only first degree invariant is $I_{1 ; e, e}=\operatorname{Tr}(\rho)$, same as with a single subsystem. (b) In the second degree we obtain a new invariant, $I_{2 ;(12), e}$ (c) There are several new third degree invariants, including the topologically distinct $I_{3 ;(123),(123)}$ and $I_{3 ;(123),(321)}$.

\section{Pure states}

If the state $\rho$ is pure, the diagrammatic structure of the LU invariants simplifies considerably, and many of the diagrams break up into unconnected sub-diagrams. Furthermore, in the case of bipartite pure states, we may apply the Schmidt decomposition and introduce graphical rewrite rules to show that these invariants reduce to polynomials of the Schmidt coefficients.

Theorem 22 (Bipartite pure states). Applying the diagrammatic Schmidt decomposition presented in Corollary 6 to the bipartite invariant diagrams in Figure 6 , we can see that the 
unitaries $U, V$ and the dimension changers $Q$ always cancel, and the invariant diagrams break up into mutually disjoint loops corresponding to sums of even powers of the Schmidt coefficients $\left\{\sigma_{i}\right\}_{i=0}^{d-1}$. Hence, the only independent invariants we obtain are of the form

$$
J_{k}:=I_{k ;(12 \cdots k), e}=\sum_{i} \sigma_{i}^{2 k} \quad \text { for } \quad k \in\{1,2, \ldots\} .
$$

In Figure 7 we present this process for the invariant

$$
I_{3 ;(123),(12)}=\left(\sum_{k} \sigma_{k}^{2}\right)\left(\sum_{k} \sigma_{k}^{4}\right)=I_{1 ; e, e} I_{2 ;(12), e}=J_{1} J_{2} .
$$
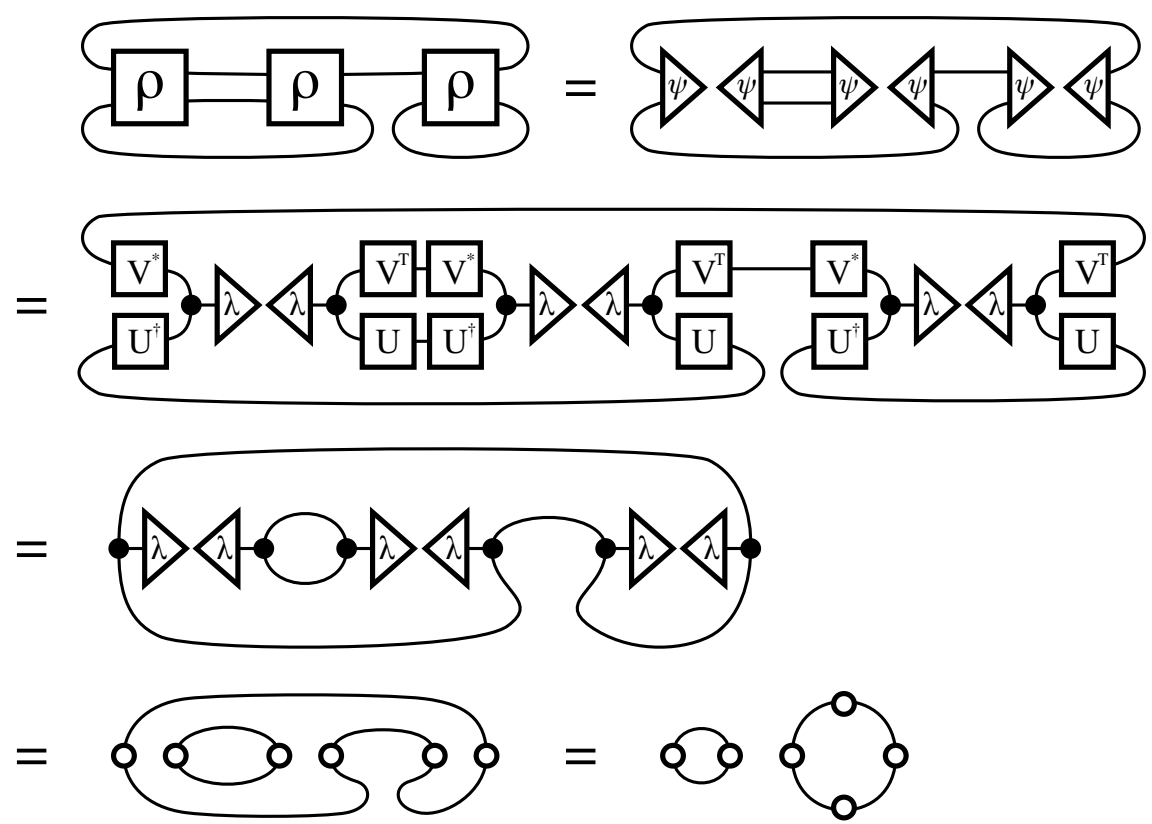

FIG. 7. Invariant $I_{3 ;(123),(12)}$ evaluated for a pure bipartite state $\rho=|\psi\rangle\langle\psi|$ using the diagrammatic Schmidt decomposition. The unitaries $U$ and $V$ (and possible dimension changers $Q$ ) cancel, and one is left with two disjoint loops, on which the blank circles denote diagonal tensors with the Schmidt coefficients $\left\{\sigma_{i}\right\}_{i=0}^{d-1}$ on the diagonal.

Since the $d$ Schmidt coefficients themselves (by construction) form a complete set of bipartite LU invariants, we should be able to express them as functions of $\left\{J_{i}\right\}_{i=1}^{d}$. This is accomplished in principle by solving the following system of polynomial equations:

$$
\begin{gathered}
\sum_{i} \sigma_{i}^{2}=J_{1}, \\
\sum_{i} \sigma_{i}^{4}=J_{2}, \\
\vdots=\vdots \\
\sum_{i} \sigma_{i}^{2 d}=J_{d} .
\end{gathered}
$$


Example 23 (Pure state of two qubits). Given a pure two-qubit state

$$
|\psi\rangle=\sum_{i j} \alpha^{i j}|i j\rangle,
$$

we wish to compute its LU invariants. Since it has two Schmidt coefficients, we expect to find two independent invariants. The first one, $J_{1}$, corresponds to the squared norm of the state, and is thus trivially invariant under unitary transformations of $|\psi\rangle$ :

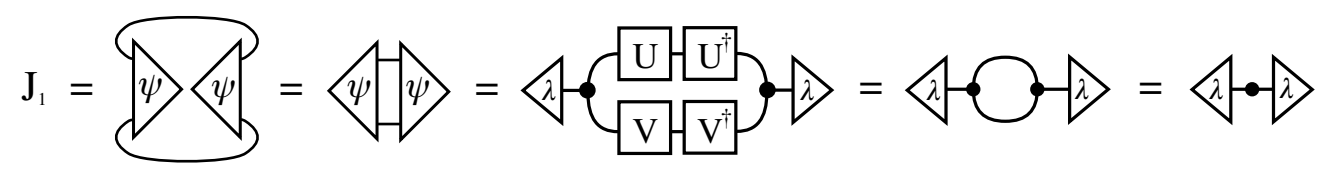

The second independent invariant, $J_{2}$, doesn't have as simple an interpretation:

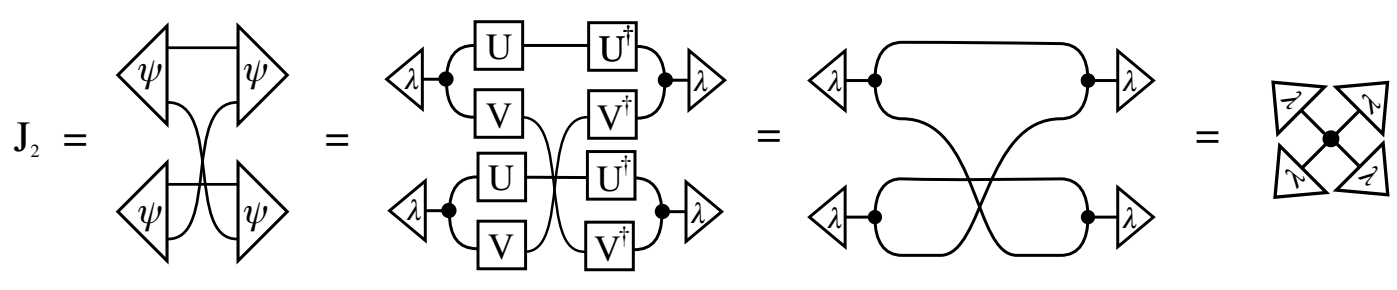

The diagrams correspond to the equations

$$
\begin{aligned}
& J_{1}=\sum_{i j} \alpha^{i j} \alpha_{i j}^{*}=\sigma_{0}^{2}+\sigma_{1}^{2}, \\
& J_{2}=\sum_{i j k l} \alpha^{i j} \alpha^{k l} \alpha_{i l}^{*} \alpha_{k j}^{*}=\sigma_{0}^{4}+\sigma_{1}^{4},
\end{aligned}
$$

with the solution

$$
\sigma_{0,1}^{2}=\frac{1}{2}\left(J_{1} \pm \sqrt{2 J_{2}-J_{1}^{2}}\right),
$$

which yields $J_{2} \leq J_{1}^{2} \leq 2 J_{2}$. For a normalized state $\left(J_{1}=1\right)$, it can be shown that the invariant $J_{2}$ can be expressed as

$$
J_{2}=1-2\left|\alpha_{00} \alpha_{11}-\alpha_{01} \alpha_{10}\right|^{2},
$$

where $\alpha_{00} \alpha_{11}-\alpha_{01} \alpha_{10}$ is simply the determinant of the coefficient matrix $\alpha$, which is non-zero iff $|\psi\rangle$ represents an entangled state.

$J_{1}$ and $J_{2}$ are the only algebraically independent LU invariants of a pure two-qubit system. Any polynomial function of such invariants is also a polynomial invariant. In this fashion, it is a remarkable feature that functions of $J_{1}$ and $J_{2}$ are all that is needed to express any local unitary invariant of two-qubit pure states. This elementary result follows from a much more powerful and general result in classical invariant theory, a proof by Hilbert that the ring of polynomial invariants is finitely generated [32]. This corresponds to freely generated linear sums and products of $J_{1}, J_{2}$, e.g. the ring $\left\{J_{1}, J_{2},(\mathbb{R},+, \cdot)\right\}$. Any minimal complete set of invariants that can freely generate the full ring are called fundamental invariants. 


\section{INVARIANTS, ENTROPIES AND ENTANGLEMENT}

Here we focus on expressing Rényi entropies in terms of the invariants we have found tensor contractions for in the previous sections. The Rényi entropy has many uses in condensed matter physics (see for example $[35,36]$ ) and recently has been given an interesting physical interpretation [37]. We will recall the definition as

Definition 24 (Rényi entropy [38]). The Rényi entropy of order $\alpha$ is defined as

$$
S_{\alpha}:=\frac{1}{1-\alpha} \ln \operatorname{Tr}\left(\rho^{\alpha}\right) .
$$

In the limit $\alpha \rightarrow 1$ we obtain

$$
\lim _{\alpha \rightarrow 1} S_{\alpha}=-\operatorname{Tr}(\rho \ln \rho),
$$

which is the von Neumann entropy.

Here we note that terms such as $\operatorname{Tr}\left(\rho^{\alpha}\right)$ are in correspondence with the tensor contractions evaluating to invariants which we have already found. To explain how we can contract tensor networks to evaluate Rényi entropies for counting $\alpha>1$, we will close the paper with a specific example, though the procedure we describe is general. We will focus explicitly on the invariants of a bipartition of a 5-party qubit state $|\psi\rangle \in \mathbb{C}^{2} \otimes \mathbb{C}^{2} \otimes \mathbb{C}^{2} \otimes \mathbb{C}^{2} \otimes \mathbb{C}^{2}$. We first recall that we can factor any state into a MPS; in the case of our example, this yields the following graphical depiction.

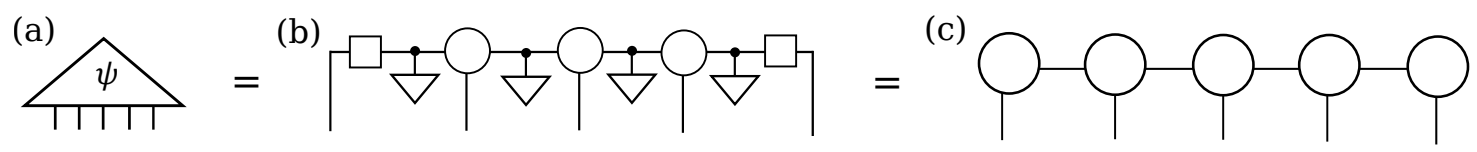

Here (a) is the original state, (b) is our factorization in terms of COPY-tensors, and (c) recovers the familiar MPS representation, as explained in Section 4. The method to evaluate Rényi entropies by tensor contraction works generally by first grouping the legs of any tensor network state into a bipartition, and to consider the correlations between the two halves. In the present example, we group the two top legs $(A)$ and the other three legs $(B)$ and then apply the graphical SVD:

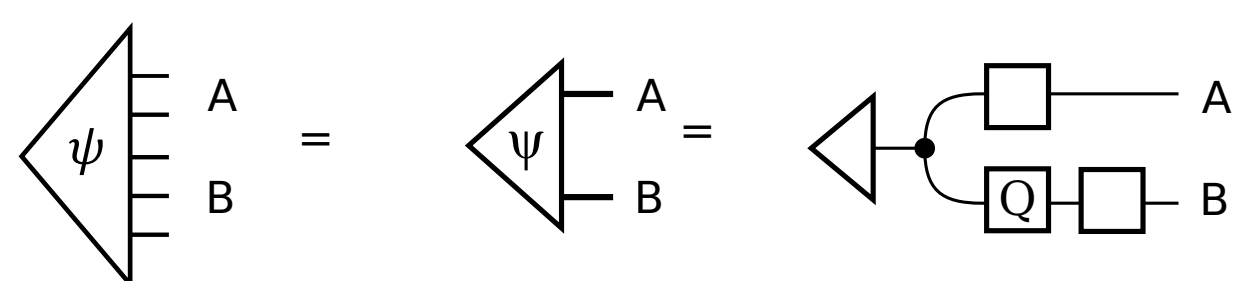

After tracing out system $B$, we are concerned with a four dimensional space $\mathbb{C}^{2} \otimes \mathbb{C}^{2}$. From the Cayley-Hamilton theorem

$$
\operatorname{Tr}\left(\rho^{4}\right)+a \operatorname{Tr}\left(\rho^{3}\right)+b \operatorname{Tr}\left(\rho^{2}\right)+c=0
$$

for some values of $a, b, c$. We hence conclude that all information we can expect to find can be obtained by evaluating tensor contractions for $\operatorname{Tr}\left(\rho^{n}\right)$ for $n=2,3,4$ as given in the 
section on invariants. As an example, to evaluate $\operatorname{Tr}\left(\rho^{3}\right)$ :

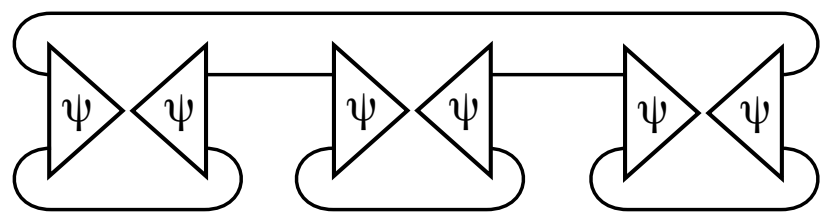

We can evaluate this contraction using a tensor network numerical algorithms package. By writing it in terms of the graphical SVD and applying the rewrite rules developed in the present work, we arrive at

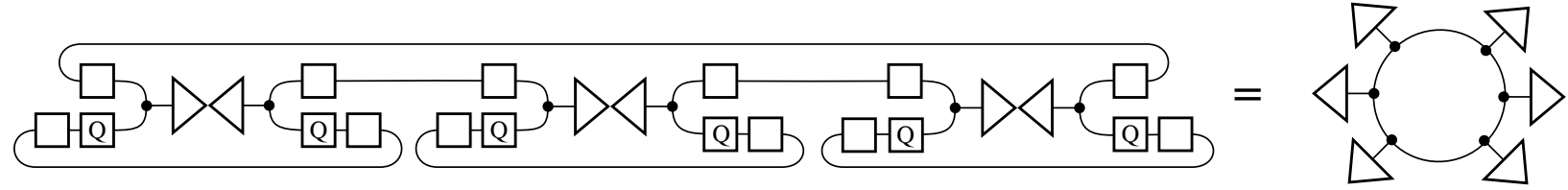

This illustrates that the network reduces to an expression in terms of the singular values of the pure state. If the singular values of the original state are given as $\left(\sigma_{1}, \sigma_{2}, \sigma_{3}, \sigma_{4}\right)=$ : $\left(\sqrt{p}_{1}, \sqrt{p}_{2}, \sqrt{p}_{3}, \sqrt{p}_{4}\right)$, then the expression evaluates to

$$
I_{3 ;(123), e}=\sigma_{1}^{6}+\sigma_{2}^{6}+\sigma_{3}^{6}+\sigma_{4}^{6}=p_{1}^{3}+p_{2}^{3}+p_{3}^{3}+p_{4}^{3}
$$

where the eigenvalues $p_{i}$ on the right are the probabilities of measuring the reduced system in the $i$ th eigenstate. We then express the Rényi entropy with $\alpha=3$ as $S_{3}=-\frac{1}{2} \ln \left(I_{3 ;(123), e}\right)$. Other quantities can be similarly calculated, resulting in the following identical relation for the Rényi entropies

$$
\exp \left(-3 S_{4}\right)+a \exp \left(-2 S_{3}\right)+b \exp \left(-S_{2}\right)+c=0
$$

which is an alternative expression for (56) in terms of the $S_{\alpha}$ from Definition 24.

\section{CONCLUSION}

We have developed a graphical method for expressing a complete polynomial basis for the local unitary invariants of any finite-dimensional quantum system. Using the diagrammatic SVD, we have shown that for pure bipartite systems these contractions can be expressed in terms of manifestly invariant singular values. These methods seem to provide new conceptual insight to understanding quantities such as entropies which are expressed as holomorphic functions of the invariants. By connecting invariant theory with tensor network states, we hope that this work leads to a better understanding of how entropies and entanglement measures can be calculated for specific models, and ultimately to a better understanding of invariants in various higher-dimensional geometries as well.

\section{ACKNOWLEDGMENTS}

We thank Markus Grassl, Ann Kallin, Jason Morton, Nilhan Gurkan and Francesco Vaccarino. VB visited the Centre for Quantum Technologies (CQT, Singapore), ML visited 
Oxford University and JB visited the Perimeter Institute for Theoretical Physics as well as the Institute for Quantum Computing during part of this work.

[1] Markus Grassl, Martin Rötteler, and Thomas Beth, "Computing local invariants of quantumbit systems," Phys. Rev. A 58, 1833-1839 (1998), arXiv:quant-ph/9712040v2.

[2] Yuriy Makhlin, "Nonlocal properties of two-qubit gates and mixed states, and the optimization of quantum computations," Quantum Information Processing 1, 243-252 (2002), arXiv:quant-ph/0002045v2.

[3] E.M. Rains, "Polynomial Invariants of Quantum Codes," IEEE Trans. Information Theory 46, 54-59 (2000),

[4] Anthony Sudbery, "On local invariants of pure three-qubit states," J. Phys. A: Math. Gen. 34, 643 (2001).

[5] M. S. Leifer, N. Linden, and A. Winter, "Measuring polynomial invariants of multiparty quantum states," Phys. Rev. A 69, 052304 (2004), arXiv:quant-ph/0308008v2.

[6] M. S. Williamson, M. Ericsson, M. Johansson, E. Sjöqvist, A. Sudbery, V. Vedral, and W. K. Wootters, "Geometric local invariants and pure three-qubit states," Phys. Rev. A 83, 062308 (2011), arXiv:1102.4222.

[7] B. Kraus and J. I. Cirac, "Optimal creation of entanglement using a two-qubit gate," Phys. Rev. A 63, 062309 (2001).

[8] Laura Koponen, Ville Bergholm, and Martti M. Salomaa, "A discrete local invariant for quantum gates," Quant. Inf. and Comp. 6, 58 (2006), arXiv:quant-ph/0503141.

[9] Roger Penrose, "Applications of negative dimensional tensors," in Combinatorial Mathematics and its Applications, edited by D. Welsh (Academic Press, New York, 1971) pp. 221-244.

[10] J. D. Biamonte, S. R. Clark, and D. Jaksch, "Categorical tensor network states," AIP Advances 1, 042172 (2011), arXiv:1012.0531.

[11] V. Bergholm and J. D. Biamonte, "Categorical quantum circuits," J. Phys. A: Math. Theor. 44, 245304 (2011), arXiv:1010.4840.

[12] S. J. Denny, J. D. Biamonte, D. Jaksch, and S. R. Clark, "Algebraically contractible topological tensor network states," J. Phys. A: Math. Theor. 45, 015309 (2012), arXiv:1108.0888.

[13] U. Schollwöck, "The density-matrix renormalization group in the age of matrix product states," Annals of Physics 326, 96-192 (2011), arXiv:1008.3477.

[14] F. Verstraete, V. Murg, and J.I. Cirac, "Matrix product states, projected entangled pair states, and variational renormalization group methods for quantum spin systems," Advances in Physics 57, 143-224 (2008).

[15] J. Ignacio Cirac and Frank Verstraete, "Renormalization and tensor product states in spin chains and lattices," J. Phys. A: Math. Theor. 42, 504004 (2009).

[16] G. Vidal, "Efficient classical simulation of slightly entangled quantum computations," Physical Review Letters 91, 147902 (2003), arXiv:quant-ph/0301063.

[17] D. Perez-Garcia, F. Verstraete, M. M. Wolf, and J. I. Cirac, "Matrix product state representations," Quant. Inf. and Comp. 7, 401 (2007), arXiv:quant-ph/0608197.

[18] Andrew Critch and Jason Morton, "Polynomial constraints on representing entangled qubits as matrix product states," (2012), arXiv:1210.2812.

[19] Jacob Turner and Jason Morton, "Some effective results on the invariant theory of tensor networks," (2013), arXiv:1310.0370. 
[20] T. H. Johnson, S. R. Clark, and D. Jaksch, "Dynamical simulations of classical stochastic systems using matrix product states," Phys. Rev. E 82, 036702 (2010), arXiv:1006.2639.

[21] S. Singh and G. Vidal, "Tensor network states and algorithms in the presence of a global SU(2) symmetry," Phys. Rev. B 86, 195114 (2012), arXiv:1208.3919.

[22] D. Gross, J. Eisert, N. Schuch, and D. Perez-Garcia, "Measurement-based quantum computation beyond the one-way model," Phys. Rev. A 76, 052315 (2007), arXiv:0706.3401.

[23] Yves Lafont, "Penrose diagrams and 2-dimensional rewriting," in Applications of Categories in Computer Science, London Mathematical Society Lecture Note Series, Vol. 177, edited by M.P. Fourman, P.T. Johnstone, and A.M. Pitts (Cambridge University Press, 1992) pp. 191-201.

[24] Yves Lafont, "Equational reasoning with 2-dimensional diagrams," in Term Rewriting, Lecture Notes in Computer Science, Vol. 909 (Springer-Verlag, 1995) pp. 170-195.

[25] Yves Lafont, "Towards an algebraic theory of Boolean circuits," Journal of Pure and Applied Algebra 184, 257-310 (2003).

[26] J. C. Baez and M. Stay, "Physics, topology, logic and computation: A Rosetta stone," in New Structures for Physics, Lecture Notes in Physics, Vol. 813 (Springer Berlin / Heidelberg, 2011) pp. 95-172, arXiv:0903.0340.

[27] John C. Baez and Aaron D. Lauda, "A prehistory of n-categorical physics," in Deep Beauty: Understanding the Quantum World Through Mathematical Innovation, edited by Hans Halvorson (Cambridge University Press, 2011) pp. 13-128, arXiv:0908.2469.

[28] C. J. Wood, J. D. Biamonte, and D. G. Cory, "Tensor networks and graphical calculus for open quantum systems," (2011), arXiv:1111.6950.

[29] S. Meznaric and J. Biamonte, "Tensor networks for entanglement evolution," to appear in Adv. Chem. Phys. (2012), arXiv:1204.3599.

[30] Jason Morton and Jacob Biamonte, "Undecidability in tensor network states," Phys. Rev. A 86, 030301 (2012), arXiv:1205.3315.

[31] G. Evenbly and G. Vidal, "Tensor network states and geometry," Journal of Statistical Physics 145, 891-918 (2011), arXiv:1106.1082.

[32] David Hilbert, Theory of Algebraic Invariants (Cambridge University Press, 1993).

[33] Claudio Procesi, "Invariant theory of $n \times n$ matrices," Advances in Mathematics 19, 306-381 (1976).

[34] Richard Brauer, "On algebras which are connected with the semisimple continuous groups," Annals of Mathematics 38, 857-872 (1937).

[35] S. T. Flammia, A. Hamma, T. L. Hughes, and X.-G. Wen, "Topological entanglement Rényi entropy and reduced density matrix structure," Phys. Rev. Lett. 103, 261601 (2009), arXiv:0909.3305.

[36] M. B. Hastings, I. González, A. B. Kallin, and R. G. Melko, "Measuring Rényi entanglement entropy in quantum Monte Carlo simulations," Phys. Rev. Lett. 104, 157201 (2010), arXiv:1001.2335.

[37] J. C. Baez, "Rényi entropy and free energy," (2011), arXiv:1102.2098.

[38] Alfréd Rényi, "On measures of entropy and information," in Fourth Berkeley Symposium on Mathematical Statistics and Probability (1961) pp. 547-561. 